ISSN 2078-3744. Вісник Львів. ун-ту. Серія мех.-мат. 2018. Випуск 86. С. 45-50

Visnyk of the Lviv Univ. Series Mech. Math. 2018. Issue 86. P. 45-50

http://publications.lnu.edu.ua/bulletins/index.php/mmf

doi: http://dx.doi.org/10.30970/vmm.2018.86.045-050

УДК 511.3

\title{
SIMULTANEOUS APPROXIMATION OF VALUES OF WEIERSTRASS AND JACOBI ELLIPTIC FUNCTIONS IN THE PERIODS AND ALGEBRAIC POINT
}

\author{
Dedicated to the 60th birthday of M. M. Zarichnyi
}

\author{
Yaroslav KHOLYAVKA, Olga MYLYO \\ Ivan Franko National University of Lviv, \\ Universytetska Str., 1, 79000, Lviv, Ukraine \\ e-mails:ya_khol@franko.lviv.ua, \\ olga.mylyo@gmail.com
}

Let $\wp(z)$, sn $z$ be algebraically independent Weierstrass and Jacobi elliptic functions with algebraic invariants and algebraic elliptic module, $\left(2 \omega_{1}, 2 \omega_{3}\right)$ and $\left(4 K, 2 i K^{\prime}\right)$ be the main periods of $\wp(z)$ and $\operatorname{sn} z$ respectively, $\alpha$ be an algebraic number different from the poles of $\wp(z)$ and $\operatorname{sn} z$. We estimate from below the simultaneous approximation of $\operatorname{sn}\left(2 \omega_{1}\right), \operatorname{sn}(\alpha), \wp(4 K)$, and $\wp(\alpha)$.

Key words: simultaneous approximation, Weierstrass elliptic function, Jacobi elliptic function.

\section{INTRODUCTION}

Let $\wp(z)$ and $\operatorname{sn} z$ be the elliptic Weierstrass function and the elliptic Jacobi function, respectively. Then $\wp(z)$ satisfies the equation $\left(\wp^{\prime}(z)\right)^{2}=4 \wp^{3}(z)-g_{2} \wp(z)-g_{3}$, the numbers $g_{2}, g_{3}$ are called invariants of $\wp(z), 2 \omega_{1}, 2 \omega_{3}$ is a fixed pair of the main periods $\wp(z)$. The function $\operatorname{sn} z$ satisfies the equation $\left(\operatorname{sn}^{\prime} z\right)^{2}=\left(1-\operatorname{sn}^{2} z\right)\left(1-\varkappa^{2} \operatorname{sn}^{2} z\right)$. The number $\varkappa$ is called the elliptic module of $\operatorname{sn} z, 0<\varkappa<1$, the number $\varkappa^{\prime}=\left(1-\varkappa^{2}\right)^{1 / 2}$ is called its additional elliptic module. A pair of main the periods sn $z$ is $\left(4 K, 2 i K^{\prime}\right)$, where $K, K^{\prime}$ are complete elliptic integrals of the first kind corresponding to $\varkappa, \varkappa^{\prime}$ ([1]). In the present article we will consider algebraically independent elliptic functions $\wp(z)$ and $\operatorname{sn} z$ with

2010 Mathematics Subject Classification: 11J82

(C) Kholyavka, Ya., Mylyo, O., 2018 
algebraic $g_{2}, g_{3}$ and $\varkappa$. Let the periods $2 \omega_{1}, 4 K$ form a lattice, $2 m \omega_{1}$ and $4 m K(m \in \mathbb{Z})$ are different from the poles of $\operatorname{sn} z$ and $\wp(z)$.

For $d(P), L(P)$ denote the degree and the length of a polynomial $P$ with integer coefficients, $d(\alpha), L(\alpha)$ is the degree and the length of algebraic number $\alpha[2, \alpha$ is different from the poles of $\wp(z)$ and $\operatorname{sn} z$. Let $\xi_{i}$ be approximating algebraic numbers, $n_{i}=d\left(\xi_{i}\right)$ and $L_{i}=L\left(\xi_{i}\right)$ be their powers and lengths respectively $(i=1, \ldots, 4)$, $n=\operatorname{deg} \mathbb{Q}\left(g_{2}, g_{3}, \varkappa, \alpha, \xi_{1}, \ldots, \xi_{4}\right)$.

Theorem 1. For any algebraic numbers $\xi_{1}, \ldots, \xi_{4}$ the following inequality holds:

$$
\left|\wp(4 K)-\xi_{1}\right|+\left|\operatorname{sn}\left(2 \omega_{1}\right)-\xi_{2}\right|+\left|\wp(\alpha)-\xi_{3}\right|+\left|\operatorname{sn}(\alpha)-\xi_{4}\right|>\exp \left(-\Lambda n^{3} T^{2}\right),
$$

where

$$
T=\max \left(\frac{\ln L_{1}}{n_{1}}+\cdots+\frac{\ln L_{4}}{n_{4}}+1, \ln n\right),
$$

$\Lambda>0$ is a constant that depends only on $g_{2}, g_{3}, \varkappa$ and $\alpha$.

Similar estimates for other numbers can be found in [2]-[6].

\section{Auxiliary statements}

In the following lemma $c_{10}, \ldots, c_{15}$ stand for positive constants that are independent of $n, n_{i}, L_{i}$ and $\lambda$.

Lemma 1 ([1]). If $z, w, z+w$ are admissible values, then

$$
\wp(z+w)=\frac{1}{4}\left(\frac{\wp^{\prime}(z)-\wp^{\prime}(w)}{\wp(z)-\wp(w)}\right)^{2}-\wp(z)-\wp(w), \quad \operatorname{sn}(z+w)=\frac{\operatorname{sn} z \operatorname{sn}^{\prime} w+\operatorname{sn} w \operatorname{sn}^{\prime} z}{1-\varkappa^{2} \operatorname{sn} z^{2} \operatorname{sn} w^{2}} .
$$

Lemma 2. For each integer $m \geqslant 1$, there exist polynomials $P_{1, s, l}, P_{2, s, l}$ with the integer coefficients such that

$$
\begin{gathered}
\frac{d^{s}}{d z^{s}}\left(\left(\wp(z)^{l}\right)=P_{1, s, l}\left(g_{2}, g_{3}, \wp(z), \wp^{\prime}(z)\right), \quad \frac{d^{s}}{d z^{s}}\left((\operatorname{sn} z)^{l}\right)=P_{2, s, l}\left(\varkappa^{2}, \operatorname{sn} z, \operatorname{sn}^{\prime} z\right),\right. \\
\operatorname{deg} P_{i, s, l} \leqslant c_{1}(s+l), \quad L\left(P_{i, s, l}\right) \leqslant \exp \left(c_{2} s \log (s+l)\right), \quad i=1,2 .
\end{gathered}
$$

Lemma 3. For each integer $m \geqslant 1$, there exist polynomials with the integer coefficients $P_{1, m}, P_{2, m}, Q_{1, m}, Q_{2, m}$ such that

$$
\wp(m z)=\frac{P_{1, m}\left(\wp(z), g_{2}, g_{3}\right)}{Q_{1, m}\left(\wp(z), g_{2}, g_{3}\right)}, \quad \text { sn } m z=\frac{P_{2, m}\left(\operatorname{sn} z, \mathrm{sn}^{\prime} z\right)}{Q_{2, m}\left(\varkappa^{2}, \operatorname{sn} z\right)},
$$

where $L\left(P_{i, m}\right), L\left(Q_{i, m}\right) \leqslant \exp \left(c_{3} m^{2}\right), \operatorname{deg} P_{i, m}, \operatorname{deg} Q_{i, m} \leqslant m^{2}, i=1,2$.

The proof of Lemma 2 and Lemma 3 for the function $\wp(z)$ is, for example, in [1], [2], [8], and proof for $\operatorname{sn} z$ is similar to the proof for $\wp(z)$.

Lemma 4 ([4]). Let $B, P \in \mathbb{N}, Q_{p, b} \in \mathbb{Z}\left[x_{1}, \ldots, x_{n}\right], 0 \leqslant b<B, 0 \leqslant p<P, L\left(Q_{p, b}\right) \leqslant L$, $\operatorname{deg}_{x_{i}} Q_{p, b} \leqslant \mathcal{N}_{i} ; \alpha_{1}, \ldots, \alpha_{n}$ be algebraic numbers, $m=\operatorname{deg} \mathbb{Q}\left(\alpha_{1}, \ldots, \alpha_{n}\right)$. If $P>m B$, then the system of linear equations

$$
\sum_{p=0}^{P-1} x_{p} Q_{p, b}\left(\alpha_{1}, \ldots, \alpha_{n}\right)=0, \quad 0 \leqslant b<B,
$$


has integer rational solutions $A_{0}, \ldots, A_{P-1}$ such that

$$
0<\max \left|A_{i}\right|<1+(L P)^{\frac{m B}{P-m B}}\left(\prod_{i=1}^{n}\left(1+\mathcal{N}_{i}\right)\left(L\left(\alpha_{i}\right)\left(1+d\left(\alpha_{i}\right)\right)\right)^{\frac{\mathcal{N}_{i}}{d\left(\alpha_{i}\right)}}\right)^{\frac{m B}{P-m B}} .
$$

We denote $|f(z)|_{D}=\sup _{|z| \leqslant D}|f(z)|$.

Lemma 5 ([5]). Let $\sigma_{1}(z)$ be the Weierstrass $\sigma$-function which corresponds to $\wp(z)$. The functions $\sigma_{1}(z)$ and $\sigma_{1}(z) \wp(z)$ is entire functions and for $M>1$

$$
\left|\sigma_{1}(z) \wp(z)\right|_{M},\left|\sigma_{1}(z)\right|_{M} \leqslant c_{4}{ }^{M^{2}} .
$$

If $\varepsilon$ is a distance from the nearest to $z_{0}$ pole of $\operatorname{sn} z$ and $\left|z_{0}\right| \leqslant M$, then $\left|\sigma\left(z_{0}\right)\right| \geqslant \varepsilon c_{5}{ }^{-M^{2}}$.

Lemma 6. Let $\sigma_{2}(z)$ be the Weierstrass $\sigma$-function which corresponds to the function $\widetilde{\wp}(z)$ associated with $\operatorname{sn}(z)$. The functions

$$
\sigma_{2}\left((z+K) / \sqrt{e_{1}-e_{3}}\right), \quad \sigma_{2}\left((z+K) / \sqrt{e_{1}-e_{3}}\right) \operatorname{sn}(z)
$$

are entire functions and for $M>1$

$$
\left|\sigma_{2}\left((z+K) / \sqrt{e_{1}-e_{3}}\right) \operatorname{sn}(z)\right|_{|z| \leqslant M} \leqslant c_{6}{ }^{M^{2}}, \quad\left|\sigma_{2}\left((z+K) / \sqrt{e_{1}-e_{3}}\right)\right|_{|z| \leqslant M} \leqslant c_{7} M^{2} .
$$

If $\delta$ is the distance from $z_{0}$ to the nearest pole of $\operatorname{sn}(z)$ and $\left|z_{0}\right| \leqslant M_{0}$, then

$$
\left|\sigma_{2}\left(\left(z+i K^{\prime}\right) / \sqrt{e_{1}-e_{3}}\right)\right| \geqslant \delta c_{8}^{-M_{0}^{2}} .
$$

The proof of Lemma 6 is similar to the proof of Lemma 5

Lemma 7 (44). Let $R_{1}, R_{2} \in \mathbb{R}, 8<4 R_{1}<R_{2}, f(z)$ be analytic in the circle $|z| \leqslant R_{2}$, and $E$ is the set of $\mathcal{D}^{2}$ points belonging to the circle $|z| \leqslant R_{1}$ and the distance between them for each pair of points is not less than $\varepsilon, 0<\varepsilon<1$. Then

$$
|f(z)|_{|z| \leqslant R_{1}} \leqslant 2|f(z)|_{|z| \leqslant R_{2}}\left(\frac{4 R_{1}}{R_{2}}\right)^{\mathcal{D}^{2} S}+2 \mathcal{D} R_{1}^{-1}\left(\frac{33 R_{1}}{\epsilon \mathcal{D}}\right)^{\mathcal{D}^{2} S} \max _{x \in E, 0 \leqslant s \leqslant S}\left|\frac{f^{(s)}(x)}{s !}\right| .
$$

Lemma 8 ([2]). Let $\alpha_{1}, \ldots, \alpha_{n}$ be algebraic numbers, $P \in \mathbb{Z}\left[x_{1}, \ldots, x_{n}\right], \operatorname{deg}_{x_{i}} P \leqslant \mathcal{N}_{i}$, $m=\operatorname{deg} \mathbb{Q}\left(\alpha_{1}, \ldots, \alpha_{n}\right)$. If $P\left(\alpha_{1}, \ldots, \alpha_{n}\right) \not \equiv 0$, then

$$
\left|P\left(\alpha_{1}, \ldots, \alpha_{n}\right)\right| \geqslant L(P)^{1-m} \prod_{i=1}^{n} L\left(\alpha_{i}\right)^{\frac{-\mathcal{N}_{i} m}{d\left(\alpha_{i}\right)}} .
$$

Lemma 9 ([1, [7). Let $P \in \mathbb{C}\left[x_{1}, x_{2}\right], P\left(x_{1}, x_{2}\right) \not \equiv 0$, be the polynomial of degree not greater than $\mathcal{D}_{1}$ in $x_{1}$ and $\mathcal{D}_{2}$ in $x_{2}, \mathcal{D}_{1}, \mathcal{D}_{2} \geqslant 1, \wp(z)$ and $\mathrm{sn} z$ are algebraic independent elliptic functions. Then the number of zeros of $P(\wp(z), \mathrm{sn} z)$, taking into account their multiplicity, for $|z|<K$ does not exceed $c_{9} K^{2}\left(\mathcal{D}_{1}+\mathcal{D}_{2}\right)$.

\section{Proof of Theorem 1}

The proof of Theorem 1 is based on the second Gelfond's method [7, 8]. Suppose that for a sufficiently large $\lambda \in \mathbb{N}$ we have

$$
\left|\wp(4 K)-\xi_{1}\right|+\left|\operatorname{sn}\left(2 \omega_{1}\right)-\xi_{2}\right|+\left|\wp(\alpha)-\xi_{3}\right|+\left|\operatorname{sn}(\alpha)-\xi_{4}\right|<\exp \left(-\lambda^{7} n^{3} T^{2}\right) .
$$


We denote

$$
N^{2}=\left[\lambda^{3} n T\right], \quad S=L=\left[N^{2} \ln \lambda\right]
$$

Define a function

$$
F(z)=\sum_{l_{1}=0}^{L} \sum_{l_{2}=0}^{L} C_{l_{1}, l_{2}} \wp^{l_{1}}(z) \operatorname{sn}^{l_{2}} z, \quad C_{l_{1}, l_{2}}=\sum_{\tau=1}^{n} C_{l_{1}, l_{2}, \tau} \zeta_{\tau}, C_{l_{1}, l_{2}, \tau} \in \mathbb{Z},
$$

where $\zeta_{\tau}$ are generating elements of $\mathbb{Q}\left(g_{2}, g_{3}, \varkappa, \alpha, \xi_{1}, \ldots, \xi_{4}\right)$. As in [8], we denote $\varphi_{1}(z)=$ $\wp\left(z+\omega_{1}\right), \varphi_{2,1}(z)=\operatorname{sn}\left(z+\frac{K}{2}\right), \varphi_{2,2}(w)=\operatorname{sn}\left(w+\frac{3 K}{2}\right)$. Then from the Lemma 1

$$
\begin{gathered}
\wp(z+w)=\frac{1}{4}\left(\frac{\varphi_{1}^{\prime}(z)-\varphi_{1}^{\prime}(w)}{\varphi_{1}(z)-\varphi_{1}(w)}\right)^{2}-\varphi_{1}(z)-\varphi_{1}(w)=\frac{\Lambda_{1,1}(z, w)}{\Lambda_{1,2}(z, w)}, \\
\operatorname{sn}(z+w)=\frac{\varphi_{2,1}(z) \varphi_{2,2}^{\prime}(w)+\varphi_{2,2}(w) \varphi_{2,1}^{\prime}(z)}{1-\varkappa^{2} \varphi_{2,1}^{2}(z) \varphi_{2,2}^{2}(w)}=\frac{\Lambda_{2,1}(z, w)}{\Lambda_{2,2}(z, w)} .
\end{gathered}
$$

From (6), (7) and Lemma 2 it follows that there exist polynomials $G_{i, s, k, l}(z)$ such that

$$
G_{i, s, k, l}(z)=\frac{d^{s}}{d w^{s}}\left(\left.\left(\Lambda_{i, 1}^{k}(z, w) \Lambda_{i, 2}^{l}(z, w)\right)\right|_{w=0},\right.
$$

$\operatorname{deg} G_{i, s, k, l} \leqslant 4(k+l), \ln L\left(G_{i, s, k, l}\right) \leqslant s \ln \left(s(k+l)+c_{10}(s+k+l)\right)$.

Applying the technique of [7, [8] one can deduce from (5), (6), (7), (8) the equality

$$
\begin{gathered}
F^{(s)}(z)=\left.\frac{d^{s}}{d w^{s}}\left(\Lambda_{1,2}^{-L}(z, w) \Lambda_{2,2}^{-L}(z, w)\left(F(z+w) \Lambda_{1,2}^{L}(z, w) \Lambda_{2,2}^{L}(z, w)\right)\right)\right|_{w=0}= \\
=\left.\sum_{t=0}^{s}\left(\begin{array}{l}
s \\
t
\end{array}\right) \frac{d^{s-t}}{d w^{s-t}}\left(\Lambda_{1,2}^{-L}(z, w) \Lambda_{2,2}^{-L}(z, w)\right)\right|_{w=0} \sum_{l_{1}=0}^{L} \sum_{l_{2}=0}^{L} C_{l_{1}, l_{2}} \sum_{i=0}^{t}\left(\begin{array}{l}
t \\
i
\end{array}\right) G_{1, t-i, l_{1}, L-l_{1}}(z) \times \\
\times G_{2, i, l_{2}, L-l_{2}}(z)=\left.\sum_{t=0}^{s}\left(\begin{array}{l}
s \\
t
\end{array}\right) \frac{d^{s-t}}{d w^{s-t}}\left(\Lambda_{1,2}^{-L}(z, w) \Lambda_{2,2}^{-L}(z, w)\right)\right|_{w=0} F_{s, t}(z) .
\end{gathered}
$$

Let $\xi_{5}^{2}=4 \xi_{1}^{3}-g_{2} \xi_{1}-g_{3}, \quad \xi_{6}^{2}=\left(1-\xi_{2}^{2}\right)\left(1-\varkappa^{2} \xi_{2}^{2}\right), \quad \xi_{7}^{2}=4 \xi_{3}^{3}-g_{2} \xi_{3}-g_{3}$, $\xi_{8}^{2}=\left(1-\xi_{4}^{2}\right)\left(1-\varkappa^{2} \xi_{4}^{2}\right)$. Applying Lemma 3 , denote by $F_{s, n_{1}, n_{2}}\left(\xi_{1}, \ldots, \xi_{8}\right)$ and $F_{s, t, n_{1}, n_{2}}\left(\xi_{1}, \ldots, \xi_{8}\right)$ the expressions obtained from $F^{(s)}\left(4 n_{1} K+2 n_{2} \omega_{1}+\alpha\right)$ and $F_{s, t}\left(4 n_{1} K+2 n_{2} \omega_{1}+\alpha\right)$ by the substitution $\wp(4 K), \operatorname{sn}\left(2 \omega_{1}\right), \wp(\alpha), \operatorname{sn}(\alpha), \wp^{\prime}(4 K)$, $\operatorname{sn}^{\prime}\left(2 \omega_{1}\right), \wp^{\prime}(\alpha), \operatorname{sn}^{\prime}(\alpha)$ on $\xi_{1}, \ldots, \xi_{8}$. Consider $F_{s, t, n_{1}, n_{2}}\left(\xi_{1}, \ldots, \xi_{8}\right)$ for $1 \leqslant n_{1}, n_{2} \leqslant N$, $0 \leqslant t \leqslant s \leqslant S$ as $N^{2} S$ of linear forms of $n L^{2}$ variables $C_{l_{1}, l_{2}, \tau}$. Applying Lemma 4, we choose $C_{l_{1}, l_{2}, \tau}$ not all equal to zero such that for $1 \leqslant n_{1}, n_{2} \leqslant N, 0 \leqslant t \leqslant s \leqslant S$

$$
F_{s, t, n_{1}, n_{2}}\left(\xi_{1}, \ldots, \xi_{8}\right)=0, \quad\left|C_{l_{1}, l_{2}, \tau}\right|<\exp \left(c_{11} \lambda^{6} \ln \lambda n^{2} T^{3}\right) .
$$

From (2), (3), (4), (10) we obtain for $1 \leqslant n_{1}, n_{2} \leqslant N, 0 \leqslant s \leqslant S$

$$
\left|F^{(s)}\left(4 n_{1} K+2 n_{2} \omega_{1}+\alpha\right)-F_{s, n_{1}, n_{2}}\left(\xi_{1}, \ldots, \xi_{8}\right)\right|<\exp \left(-\frac{1}{2} \lambda^{7} n^{2} T^{3}\right) .
$$

From (10), 11) for $1 \leqslant n_{1}, n_{2} \leqslant N, 0 \leqslant s \leqslant S$ it follows

$$
\left|F^{(s)}\left(4 n_{1} K+2 n_{2} \omega_{1}+\alpha\right)\right|<\exp \left(-\frac{1}{2} \lambda^{7} n^{2} T^{3}\right) .
$$


We show that 12 holds for $1 \leqslant n_{1}, n_{2} \leqslant N, 0 \leqslant s \leqslant \lambda S$.

Let $H(z)=F(z) \sigma_{1}^{L}(z) \sigma_{2}^{L}\left((z+K) / \sqrt{e_{1}-e_{3}}\right)$. We will select the least $r \in \mathbb{N}$ such that $r>32 N\left(|K|+\left|\omega_{1}\right|+1\right), R=12 r$. From (2), (4), (5), (10) and Lemma 5 it follows

$$
|H(z)|_{|z| \leqslant R}<\exp \left(-\lambda^{6} \ln \lambda n^{2} T^{3}\right) .
$$

From 13 and Lemma 7 we obtain for $0 \leqslant s \leqslant \lambda S$

$$
\left|H^{(s)}(z)\right|_{|z| \leqslant r}<\exp \left(-\frac{1}{2} \lambda^{6} \ln \lambda T^{2} \ln T\right) .
$$

From Lemma 6 for a sufficiently small $\varepsilon$ in the $\varepsilon$-neighborhood of points $4 n_{1} K+\alpha$ function $\sigma_{2}\left((z+K) / \sqrt{e_{1}-e_{3}}\right)$ and $\varepsilon$-neighborhood of the points $2 n_{2} \omega_{1}+\alpha$ the function $\sigma_{1}(z)$ has no zeros, thus for $\left|n_{1}\right|,\left|n_{2}\right| \leqslant 32 N$ we see that

$$
\begin{gathered}
\left|\sigma_{1}(z)\right|_{z \in V\left(\varepsilon, 4 n_{1} K+2 n_{2} \omega_{1}+\alpha\right)}>\exp \left(-c_{12} \lambda^{5} \ln \lambda n^{2} T^{3}\right), \\
\left|\sigma_{2}\left((z+K) / \sqrt{e_{1}-e_{3}}\right)\right|_{z \in V\left(\varepsilon, 4 n_{1} K+2 n_{2} \omega_{1}+\alpha\right)}>\exp \left(-c_{13} \lambda^{5} \ln \lambda n^{2} T^{3}\right) .
\end{gathered}
$$

The conditions $(13)-(16)$ imply that for $1 \leqslant n_{1}, n_{2} \leqslant N, 0 \leqslant s \leqslant \lambda S$

$$
\left|F^{(s)}\left(4 n_{1} K_{1}+4 n_{2} K_{2}+\alpha\right)\right|<\exp \left(-\frac{1}{3} \lambda^{6} \ln \lambda n^{2} T^{3}\right) .
$$

From 11] and (17) for $1 \leqslant n_{1}, n_{2} \leqslant N$ and $0 \leqslant s \leqslant \lambda S$ it follows

$$
\left|F_{s, n_{1}, n_{2}}\left(\xi_{1}, \ldots, \xi_{8}\right)\right|<\exp \left(-\frac{1}{4} \lambda^{6} \ln \lambda n^{2} T^{3}\right) .
$$

Considering $\left.F_{s, t, n_{1}, n_{2}}\left(\xi_{1}, \ldots, \xi_{8}\right)\right), 0 \leqslant t \leqslant s \leqslant \lambda S, 1 \leqslant n_{1}, n_{2} \leqslant N$, as the value of the corresponding polynomial in the algebraic points, from Lemma 8 we obtain for $\left.F_{s, t, n_{1}, n_{2}}\left(\xi_{1}, \ldots, \xi_{8}\right)\right) \neq 0$ the inequality

$$
\left.\mid F_{s, t, n_{1}, n_{2}}\left(\xi_{1}, \ldots, \xi_{8}\right)\right) \mid>\exp \left(-\lambda^{5} \ln \lambda n^{2} T^{3}\right) .
$$

From (9), (19) we obtain for $1 \leqslant n_{1}, n_{2} \leqslant N, 0 \leqslant s \leqslant \lambda S$

$$
\left.\mid F_{s, n_{1}, n_{2}}\left(\xi_{1}, \ldots, \xi_{8}\right)\right) \mid>\exp \left(-2 \lambda^{5} \ln \lambda n^{2} T^{3}\right) .
$$

Since (18) and (20) are contradictory,

$\left.F_{s, t, n_{1}, n_{2}}\left(\xi_{1}, \ldots, \xi_{8}\right)\right)=0$ for $1 \leqslant n_{1}, n_{2} \leqslant N, 0 \leqslant t \leqslant s \leqslant \lambda S$. Then for $1 \leqslant n_{1}, n_{2} \leqslant$ $N, 0 \leqslant s \leqslant \lambda S$

$$
F_{s, n_{1}, n_{2}}\left(\xi_{1}, \ldots, \xi_{8}\right)=0 .
$$

From (21) it follows that the polynomial $F(z)$ has at least $c_{14} \lambda^{7} \ln \lambda n^{2} T^{2}$ zeros (taking into account multiplicity), but according to Lemma 9 the number of zeros can be at most $c_{15} \lambda^{6} \ln \lambda n^{2} T^{2}$, therefore for sufficiently large $\lambda \in \mathbb{N}$ assumption (3) leads to the contradiction which proves the theorem.

\section{REFERENCES}

1. D. F. Lawden, Elliptic functions and applications, Springer-Verlag, Berlin, 1989.

2. Н. И. Фельдман, Седъмал проблема Гилъберта, Изд-во МГУ, Москва, 1982.

3. N. I. Fel'dman, Yu. V. Nesterenko, Transcendental Numbers, Springer-Verlag, Berlin, 1998.

4. E. Reyssat, Approximation algébrique de nombres lies aux fonctions elliptique et exp, Bull. Soc. Math. France. 108 (1980), 47-79. 
5. D. Masser, Elliptic functions and transcendence, Springer-Verlag, Berlin, 1975.

6. О. Мильо, Я. Холявка, Сумісні наближення значенъ еліптичних функиій Вейєритрасса та Якобі, Вісник Львів. ун-ту. Сер. прикл. мат. інформ. 22 (2014), 85-91.

7. Ю. В. Нестеренко, О мере алгебрачческой независимости значений эллиптической функиии, Изв. РАН. Сер. матем. 59 (1995), nо. 4, 155-178; English version: Yu. V. Nesterenko, On the measure of algebraic independence of values of an elliptic function, Izv. Math. 59 (1995), no. 4, 815-838. DOI: 10.1070/IM1995v059n04ABEH000035

8. G. V. Chudnovsky, Algebraic independence of the values of elliptic functions at algebraic points; Elliptic analogue of the Lindemann-Weierschtrass theorem, Invent. Math. 61 (1980), no. 3, 267-290. DOI: $10.1007 /$ BF01390068

Стаття: надійшла до редколегї̈ 28.01.2019

доопрацвована 11.02.2019

прийнята до друку 18.02.2019

\section{СУМІСНІ НАБЛИЖЕННЯ ЗНАЧЕНЬ ЕЛІПТИЧНИХ ФУНКЦІЙ ВЕЙЄРШТРАССА ТА ЯКОБІ В ПЕРІОДАХ І АЛГЕБРИЧНІЙ ТОЧЦІ}

\section{Ярослав ХОЛЯВКА, Ольга МИЛЬО}

Лъвівсъкий націоналъний університет імені Івана Франка, вул. Університетсъка, 1, 79000, Львів

e-mails:ya_khol@franko.lviv.ua, olga.mylyo@gmail.com

Нехай $\wp(z), \operatorname{sn} z$ - алгебрично незалежні еліптичні функції Вейєрштрасса та Якобі з алгебричними інваріантами й еліптичним модулем, $\left(2 \omega_{1}, 2 \omega_{3}\right)$ i $\left(4 K, 2 i K^{\prime}\right)$ - пари основних періодів $\wp(z)$ та sn $z, \alpha$ - довільне алгебричне число, відмінне від полюсів $\wp(z)$ i sn $z$. Отримано оцінку сумісного наближення $\operatorname{sn}\left(2 \omega_{1}\right), \operatorname{sn}(\alpha), \wp(4 K)$ та $\wp(\alpha)$.

Ключові слова: сумісні наближення, еліптична функція Вейєрштрасса, еліптична функція Якобі. 